\title{
THE RECOGNITION PROBLEM FOR TOPOLOGICAL MANIFOLDS: A SURVEY
}

\author{
By Dušan Repovš
}

\begin{abstract}
This is a survey of recent work on the problem of recognizing topological manifolds among topological spaces, including the results of J.L. Bryant, S.C. Ferry, W. Mio and S. Weinberger in higher dimensions, and M.V. Brahm, R.J. Daverman and D. Repovš in dimension three.
\end{abstract}

\section{Introduction}

The definition of a topological $n$-manifold $M(n \in \mathbf{N})$ is quite simple to state - besides the separability and metrizability we require (and this is the key geometric property of manifolds) that every point $x \in M$ should possess a neighbourhood $U \subset M$ which is homeomorphic to $\mathbf{R}^{n}$. (We shall only consider closed topological manifolds $M$, i.e. $M$ is connected, compact and $\partial M=\emptyset$.) However, in practice, it is precisely the verification of the existence (or nonexistence) of such homeomorphism $h: U \rightarrow \mathbf{R}^{n}$, which is the biggest problem. So a natural question arises: Is it possible to find a characterization of topological manifolds which does not mention homeomorphisms, which is reasonably simple to state but which is also not too difficult to verify?

This is the so-called Recognitıon problem for topological manifolds, one of the most important problems of geometric topology, i.e. of its branch called Bing (or Texas) topology. In the present paper we plan to survey the most recent work on this problem, including the results due to J.L. Bryant, S.C. Ferry, W. Mio and S. Weinberger [10] in higher dimensions and M.V. Brahm [7], R.J. Daverman and D. Repovš [18] [19] in dimension three. For an account of the work done earlier see the surveys of J.W. Cannon [13] and D. Repovs [34] [35] (the survey [35] also contains an extensive bibliography on this subject). For the closely related topics - the topology of cell-like maps - the reader may wish to consult the latest survey by W.J.R. Mitchell and D. Repovš [26].

This paper is in its final form and no version of it will be submitted for publication elsewhere. Supported in part by a grant from the Ministry of Science and Technology of Republic of Slovenia. 1991 Mathematics Subject Classification. Primary: 54B15, 57N75, 57P05, Secondary: 57N10, 57N60, 57P05, 57N15

Keywords and phrases: Recognition of manifolds, cell-like resolution, generalized manifold, general position property

Received July 19, 1993. 


\section{Generalized manifolds}

Topological manifolds of dimensions 1 and 2 have very simple characterizations. For example, $S^{1}$ is the only compact, connected metric space containing at least 2 points, which is separated by every pair of its points [28], and $S^{2}$ is the only nondegenerate locally connected, connected, compact metric space which is separated by no pair of its points but is separated by each of its simple closed curves [3]. Nothing so simple is known to characterize higher dimensional manifolds.

The first question that arises in dealing with higher dimensions $(\geqq 3)$ is the following one: In which class of topological spaces do we want to detect topological manifolds? The most appropriate seems to be the class of so-called generalized manifolds. For these are the spaces which possess all the basic algebraic topology properties of manifolds, i.e. from the point of view of homology and homotopy theory they behave much like genuine manifolds (e.g. they satisfy the Poincaré duality and they are locally contractible). Also, they have all the required general topology properties - separability and metrizability. The main difference between topological $n$-manifolds and generalized $n$-manifolds is that the latter may fail to possesses sufficient general position properties (although they do possess some - cf. the work of J.J. Walsh [39] and W.J.R. Mitchell, D. Repovš and E.V. Ščepin [27]). In fact, there are examples due to R.J. Daverman and J.J. Walsh [20] of ghastly generalized manifolds, for all dimension $\geqq 3$, illustrating just how wild this class of spaces can be.

Generalized manifolds were first introduced into topology in the 1930's - one of the major motivations was the discovery by R.L. Wilder [40] that they were the proper framework to generalize classical theorems of the Jordan-Schoenflies type from dimension 2 to higher dimensions (since the examples like the Alexander horned sphere [1] makes a direct generalization impossible). Since then they have played an important role in various parts of topology, e.g. theory of transformation groups [4], theory of cell-like decompositions of manifolds [17], taming theory [12], suspensions of homology spheres [14], compactifications of open topological manifolds [8], manifold factors [15], etc.

Through the decades the definitions of a generalized manifold were changing, depending upon the particular interests of the people involved. The following is the geometric topologist's version:

Definition 2.1.. A locally compact Hausdorff space $X$ is said to be a generalized $n$-manifold $(n \in \mathrm{N})$ if $X$ satisfies the following properties:

(i) $X$ is an Euclidean neighbourhood retract (ENR), i.e. for some integer $m, X$ embeds in $\mathbf{R}^{m}$ as a retract of an open subset of $\mathbf{R}^{m}$ (equivalently, $X$ is a locally compact, finite-dimensional separable, metrizable ANR); and

(ii) $X$ is a $\mathbf{Z}$-homology $n$-manifold, i.e. for every point $x \in X$,

$$
H_{*}(X, X \backslash\{x\} ; \mathbf{Z}) \cong H_{*}\left(\mathbf{R}^{n}, \mathbf{R}^{n} \backslash\{0\} ; \mathbf{Z}\right) .
$$

Let $X$ be a generalized $n$-manifold. If $n \leqq 2$ then it follows by classical results that $X$ is a topological $n$-manifold. On the other hand, if $n \geqq 3$ then $X$ need not be a genuine $n$-manifold anymore, in fact, it may fail to possess Euclidean $n$-dimensional neighbourhoods at all points $x \in X$. Such points are called singularities of $X$ and they form the singular set $S(X)$ of $X$, i.e. $S(X)=\{x \in X \mid x$ does not have any neighbourhood in 
$X$ homeomorphic to $\mathbf{R}^{n}$ \}. Its complement, $M(X)=X \backslash S(X)$, is called the manifold set of $X$ and, if $S(X) \neq X$, it is clearly an open $n$-manifold. (It is interesting to observe that for many totally singular generalized manifolds $X$, i.e. $S(X)=X$, the singularities completely vanish upon multiplication of $X$ by the real line, i.e. $X \times \mathbf{R}$ is a genuine manifold - see [15].)

\section{Resolutions of generalized manifolds}

A resolution of an $n$-dimensional ANR $X$ is a proper, cell-like map $f: M \rightarrow X$ from a topological $n$-manifold $M$ onto $X$. It follows by classical results that if $X$ admits a resolution, $X$ must be a generalized $n$-manifold [24]. A resolution $f: M \rightarrow X$ is said to be conservative if it is one-to-one over the manifold set $M(X)$ of $X$, i.e. for every point $x \in M(X), f^{-1}(x)=$ point. It is not too difficult to show that if a generalized manifold has a resolution then it also has a conservative one.

Cell-like maps $f: M \rightarrow X$ are those whose point-preimages $f^{-1}(x)$ are cell-like sets, i.e. conti-nua with the (Borsuk) shape of a point [5]: $\operatorname{Sh}\left(f^{-1}(x)\right)=\operatorname{Sh}(\mathrm{pt}$ ). Equivalently (for finite-dimensional sets), $f^{-1}(x)$ is cell-like if it embeds into some Euclidean space $\mathbf{R}^{m}$ as a cellular set in $\mathbf{R}^{m}$, i.e. the embedding $f^{-1}(x) \hookrightarrow \mathbf{R}^{m}$ can be represented as the intersection of a properly nested decreasing sequence of closed $m$-cells [9]

$$
\mathbf{R}^{m} \supset B_{1}^{m} \supset \operatorname{int} B_{1}^{m} \supset B_{2}^{m} \supset \operatorname{int} B_{2}^{m} \supset \cdots \supset f^{-1}(x)=\bigcap_{i=1}^{\infty} B_{i}^{m} .
$$

Roughly speaking, the concept of a cell-like map is a generalization of the idea of a homeomorphism since point-preimages of homeomorphism are points whereas pointpreimages of a cell-like map only have homotopical and geometrical properties very much like a point.

Cell-like maps play an important role in topology and they have been significantly applied in solutions of several very difficult problems, e.g. the 4-dimensional Poincaré Conjecture [22]. It thus comes as no surprise that they also play a key role in the Recognition problem. (For more on topology of cell-like maps see the survey [26].)

. The strategy for attacking the Recognition problem which J.W. Cannon [13] had in mind when he proposed the problem at the 1977 AMS Summer Meeting in Seattle, consisted of two key steps: resolution and shrinkıng. More precisely, given a generalized $n$-manifold $X$, one first seeks to build a resolution of $X, f: M \rightarrow X$, by blowing up the singularities of $X$ into cell-like sets. We thus at once have the following problem:

\section{Resolution Problem. Does every generalized manifold have a resolution?}

Second, given the resolution $f: M \rightarrow X$, one considers the associated cell-like, upper semicontinuous decomposition $G_{f}=\left\{f^{-1}(x) \mid x \in X\right\}$ of $M$, consisting of the preimages of the map $f$, and tries to establish some general position properties of $X$ which would allow the controlled, simultaneous shrinking of the elements of the decomposition $G_{f}$ to arbitrary small sizes. If such a manipulation can be carried out then the classical Shrinking theorem of R.H. Bing [25] tells us that $f$ is a near-homeomorphism, i.e. $f$ can be approximated arbitrarily closely by homeomorphisms $h: M \rightarrow X$. In particular, $X$ must be homeomorphic to $M$, hence itself a topological $n$-manifold. So the other key 
question we must address is the following one:

General Position Problem. Which general position property for a fin te-dimensional ANR $X$, where $X$ is the image of a cell-like map $f: M \rightarrow X$ on an $n$-manifold $M$, implies that $f$ is a near-homeomorphism?

In this paragraph we address the first problem. The best result so far in dimensions $\geqq 5$ is the following Resolution theorem (for a complete history of previous the work on this topics see the survey [35]):

THEOREM 3.1 (F.S. Quinn [33]-[31]). Let X be a connected generalized $n$-manifold, $n \geqq 5$. Then there is an integral invariant $I(X) \in H_{0}(X ; \mathbf{Z})$ of $X$ such that:

(i) $I(X) \equiv 1 \quad(\bmod 8)$;

(ii) For every open subset $U \subset X, I(X)=I(U)$;

(iii) For every generalized m-manifold $Y, m \geqq 5, I(X \times Y)=I(X) \times I(Y)$; and

(iv) $I(X)=1$ if and only if $X$ admits a resolution.

Moreover, if $f_{\imath}: M_{\imath} \rightarrow X, i \in\{1,2\}$, are any two conservative resolutions of $X, n \geqq 4$, and $U \subset X$ is a neighbourhood of $S(X)$, then there is a homeomorphism $h: M_{1} \rightarrow M_{2}$ such that $f_{1}(x)=f_{2} h(x)$, for every point $x \in X \backslash U$.

For several years it was unknown whether Quinn's local surgery obstruction $I(X)$ can ever be nontrivial, i.e. whether there perhaps exist nonresolvable generalized manifolds. Finally, last year the following surprising results were announced:

Theorem 3.2 (J.L. Bryant, S.C. Ferry, W. Mio and S. Weinberger [10]). For every integers $n \geqq 6$ and $m \geqq 1$, and for every simply connected, closed $n$-manifold $M$, there exists a generalized $n$-manifold $X$ such that:

(i) $I(X)=m$ (hence $X$ does not admit a resolution and is totally singular); and

(ii) $X$ is homotopy equivalent to $M$.

Theorem 3.3 (J.L. Bryant, S.C. Ferry, W. Mio and S. Weinberger [10]). For every integer $n \geqq 6$, there exists a generalized $n$-manifold $X$ such that

(i) $X$ does not admit a resolution; and

(ii) $X$ us not homotopy equivalent to any topological manifold.

Essentially nothing is known in dimension 4, except for the fact that a generalized 4manifold $X$ has a resolution if and only if $X \times \mathbf{R}$ has one. This follows from the following result:

THEOREM 3.4 (F.S. Quinn [30]). Let $X$ be a generalized $n$-manifold ( $n \geqq 4)$. Then the following statements are equivalent:

(i) $X$ has a resolution;

(ii) For some $k \in \mathbf{N}, X \times \mathbf{R}^{k}$ has a resolution; and

(iii) $X \times \mathbf{R}^{2}$ is a topological $(n+2)$-manifold.

In dimension 3, the Resolution problem is entangled with the Poincaré conjecture, 
e.g. if there exist fake 3-cells it's easy to construct a nonresolvable generalized 3-manifold $X$, homotopy equivalent to $S^{3}$, with just one singularity [34]. The following is the best result so far (for earlier work see the survey [34]):

Theorem 3.5 (T.L. Thickstun [38] ). If the Poincaré conjecture is true then every generalized 3-manıfold $X$ with $\operatorname{dim} S(X)=0$ admits a (conservatıve) resolution.

Finally, in the lowest dimension $\leqq 2$, there is no problem since we've already observed that the algebraic properties of generalized $(\leqq 2)$ - manifolds are strong enough to imply also the geometric properties and hence there can be no singularities at all.

\section{General Position Properties}

Higher dimensional $(\geqq 5)$ topological manifolds possess the following simple general position property:

Definition 4.1. A metric space $X$ is said to have the disjoint disks property (DDP) if for every pair of maps $f, g: B^{2} \rightarrow X$ of the closed 2-cell $B^{2}$ into $X$ and every $\varepsilon>0$ there exist maps $f^{\prime}, g^{\prime}: B^{2} \rightarrow X$ such that $d\left(f, f^{\prime}\right)<\varepsilon, d\left(g, g^{\prime}\right)<\varepsilon$ and $f^{\prime}\left(B^{2}\right) \cap g^{\prime}\left(B^{2}\right)=\emptyset$.

It turns out that this property is also, to a large extent, characteristic for manifolds in this dimension range. This follows from the beautiful R.D. Edwards Cell-like approximation theorem:

Theorem 4.2 (R.D. Edwards [21] ). Let $M$ be a topological $n$-manifold, $n \geqq 5$, and let $f: M \rightarrow X$ be a surjective cell-like map of $M$ onto an $A N R X$. Then $X$ is a topological $n$-manifold if and only if $X$ has the DDP.

As a corollary we immediately get the solution of the Recognition problem for dimensions $\geqq 5$ :

COROLlaRY 4.3. For every $n \geqq 5$, the class of topological $n$-manifolds $\mathcal{M}_{n}$ is equal to the class of generalized n-manifolds $\mathcal{G}_{n}$ with the DDP and vanıshing Quinn's local surgery obstruction $I(X)$.

Let $X$ be any generalized $n$-manifold, $n \geqq 6$, which doesn't admit a resolution. Then by Theorem (3.1), the product $X \times T^{2}$ is also a generalized $(n+2)$-manifold without a resolution. However, by a theorem of R.J. Daverman [16], $X \times T^{2}$ has the DDP, so Theorem (3.2) implies that there exist generalized $m$-manifolds, $m \geqq 8$, which are not topological $m$-manifolds although they do possess the DDP.

In dimension 3 the solution is unfortunately not so elegant. This is hardly surprising since there is less room for moving objects apart in this dimension. Here are the appropriate versions of DDP for 3-manifolds introduced by R.J. Daverman and D. Repovš in [18] [19] (for other properties which were used earlier to solve special cases of the General position problem see the survey [34]): 
Definition 4.4. A metric space $X$ is said to have the Weak simplicial approximation property (WSAP) if for every map $f: B^{2} \rightarrow X$ of the closed 2-disk $B^{2}$ into $X$, and for every $\varepsilon>0$, there exists a map $f^{\prime}: B^{2} \rightarrow X$ such that for every $t \in B^{2}$, $d\left(f(t), f^{\prime}(t)\right)<\varepsilon$ and $f^{\prime}\left(B^{2}\right) \subset \bigcup_{i=1}^{n} D_{i}$, for some family $\left\{D_{i}\right\}_{i=1}^{3=n}$ of 1-LCC embedded 2-cells $D_{i} \subset X$.

Recall that a subset $Z \subset X$ of space $X$ is locally simply coconected (1- LCC) if every $x \in X$ and every neighbourhood $U \subset X$ of $x$, there is a neighbourhood $V \subset U$ of $x$ such that the inclusion-induced homomorphism $\Pi_{1}(V \backslash Z) \rightarrow \Pi_{1}(U \backslash Z)$ is trivial.

Definition 4.5. A metric space $X$ is said to have the Simplicial approximation property (SAP) if for every map $f: B^{2} \rightarrow X$ and every $\varepsilon>0$, there exist a map $f^{\prime}: B^{2} \rightarrow X$ and a finite topological 2-complex $K_{f^{\prime}} \subset X$ such that

(i) For every $t \in B^{2}, d\left(f(t), f^{\prime}(t)\right)<\varepsilon$;

(ii) $f^{\prime}\left(B^{2}\right) \subset K_{f^{\prime}}$; and

(iii) $X \backslash K_{f^{\prime}}$ is 1-FLG (free local fundamental group) in $X$.

$X$ is said to have the Spherical simplicial approximation property (SSAP) if the above holds when $B^{2}$ is replaced by $S^{2}$ throughout.

We define that $X \backslash K_{f^{\prime}}$ is 1-FLG in $X$ if for every $y \in K_{f^{\prime}}$ and for every sufficiently small neighbourhood $U \subset X$ of $y$, there exists another neighbourhood $V \subset U$ of $y$, such that for every connected open neighbourhood $W \subset V$ of $y$, for each nonempty component $W^{\prime} \subset W$ of $W \backslash K_{f^{\prime}}$, the inclusion-induced image of $\Pi_{1}\left(W^{\prime}\right) \rightarrow \Pi_{1}\left(U^{\prime}\right)$ is a free group on $m-1$ generators, where $U^{\prime} \subset U$ is the component of $U \backslash K_{f^{\prime}}$ containing $W^{\prime}$ and $m$ is the number of components of $\operatorname{st}(y) \backslash y$ that meet $\mathrm{Cl}\left(W^{\prime}\right)$ [29].

The definition of the 1-FLG property is admittedly a bit technical. However, note that for any finite, connected 2-complex $K$, having no local separating points and lying in a generalized 3-manifold $X$, the following are equivalent:

(i) $X \backslash K$ is 1 -FLG in $X$;

(ii) $K$ is $1-\mathrm{LCC}$ in $X$; and

(iii) Each 2-simplex of $K$ is 1-LCC in $X$ (cf. [19]).

It is easy to see that every topological $(\geqq 3)$-manifold has WSAP, SAP and SSAP. We shall need another one (its verification for 3-manifolds is less trivial [18]):

Definition 4.6. A metric space $X$ is said to have the Light map separation property (LMSP) if for every $\varepsilon>0$, every $k \in \mathbf{N}$, and every map $f: B \rightarrow X$ of a collection of $k$ standard 2-cells $B=\coprod_{i=1}^{k} B_{i}^{2}$ into $X$ such that:

(i) $N_{f} \subset$ int $B$, where $N_{f}=\left\{y \in B \mid f^{-1}(f(y)) \neq y\right\}$;

(ii) $\operatorname{dim} N_{f} \leqq 0$; and

(iii) $\operatorname{dim} Z_{f} \leqq 0$, where $Z_{f}=\left\{x \in X \mid x \in f\left(B_{i}^{2}\right) \cap f\left(B_{j}^{2}\right)\right.$ for some $\left.i \neq j\right\}$;

there exists a map $F: B \rightarrow X$ such that

(1) $d(F, f)<\varepsilon$;

(2) $F|\partial B=f| \partial B$; and

(3) For every $i \neq j, F\left(B_{i}^{2}\right) \cap F\left(B_{j}^{2}\right)=\emptyset$. 
We can now state the two main results from [19] which solve the General position problem for 3-manifods:

Theorem 4.7 (R.J. Daverman and D. Repovš [19]). A resolvable generalized 3manifold is a topological 3-manifold if and only if it possesses the SSAP.

Theorem 4.8 (R.J. Daverman and D. Repovš [19]). A resolvable generalized 3manifold $X$ is a topological 3-manifold if and only if $X$ possesses the WSAP and LMSP.

One defines the property LMSP* to be LMSP without the condition (iii). A conjecture from [18] that all 3-manifolds possess this (stronger) property was recently verified (using very technical argument) by M.V. Brahm:

THEOREM 4.9 (M.V. Brahm [7]). Every topological 3-manifold has the LMSP*.

\section{Epilogue}

Let's make a review of the major questions concerning the Recognition problem. In dimension three the Resolution problem remains open (modulo the Poincare conjecture) for generalized 3-manifolds $X$ with $\operatorname{dim} S(X) \geqq 1$ :

QUESTION 5.1. Suppose that there exist no fake cubes. Does there exist a nonresolvable generalized 3-manifold $X$ ? (Note that for such an example $X, \operatorname{dim} S(X) \geqq 1$ us necessary.)

In attemping to find a resolution, one may well wish to try the simplest unknown case (note that if $S(X)$ lies in a set $Z \subset X$ which is 1-LCC embedded in $X$ and $\operatorname{dim} Z=0$ then $S(X)=\emptyset[11])$ :

QUESTION 5.2. Suppose that there exist no fake cubes. Let $X$ be a generalized 3manifold and suppose that $S(X) \subset Z$, where $Z$ is an arc which is 1 -LCC embedded in $X$. Does $X$ admit a resolution?

Note that in order to resolve a generalized 3-manifold $X$ it suffices to find an almost $\mathbf{Z}_{2}$-acyclic resolution of $X$ :

THEOREM 5.3 (D.Repovš and R.C. Lacher [37]). Let $f: M \rightarrow X$ be a closed, monotone map from a 3-manifold $M$ onto a locally simply connected $\mathbf{Z}_{2}$-homology 3manifold $X$. Suppose that there is a 0-dimensional (possibly dense) set $Z \subset X$ such that for every point $x \in X \backslash Z, \check{H}^{1}\left(f^{-1}(x) ; \mathbf{Z}_{2}\right)=0$. Then the set $C=\left\{x \in X \mid f^{-1}(x)\right.$ is not cell-like $\}$ is locally fintte in $X$. Moreover, $X$ is a resolvable generalized 3-manifold.

Before we proceed to dimensions $\geqq 4$, we wish to ask few more questions about generalized 3-manifolds. First, consider the following result (the Dehn's lemma property and the Map separation property are another kind of general position properties of 3manifolds which were used earlier to shrink certain cell-like decompositions of 3-manifolds 
- see [36] ):

Theorem 5.4 (W. Jakobsche and D. Repovs [23]). Suppose that there exist fake cubes. Then there exists a compact homogeneous ANR $X$ with the following properties:

(1) $X$ is a generalized 3-manifold and $S(X)=X$;

(2) $X$ does not admit a resolution;

(3) $X$ has the Dehn's lemma property;

(4) $X$ has the Map separation property;

(5) $X \times S^{1}$ is homeomorphic to $S^{3} \times S^{1}$.

QUESTION 5.5. Does the example of W. Jakobsche and D. Repovš [23] also possess any of the following position properties:
(1) $L M S P(*)$
; or
(2) $(W) S A P$
; or
(3) $S S A P$ ?

The natural converse of Theorem (4.9) is still a conjecture:

Conjecture 5.6 (R.J. Daverman and D. Repovš [18]). A resolvable generalized 3-manifold $X$ is a topological 3-manifold if $X$ possesses the LMSP*.

Related to this is the following conjecture which would significantly strengthen Theorem (4.9) (for more on this conjecture and its equivalents see [6] and [7]):

ConjeCtURE 5.7. Let $f: N \rightarrow M$ be a continuous map of a 2-manifold $N$ into a 3-manifold $M$ such that $\operatorname{dim} N_{f}=0$ and $N_{f} \subset$ int $N$. Then for every contınuous function $\varepsilon:$ int $N \rightarrow(0,1]$ going to zero near $\partial N$ there exists an embedding $g: N \rightarrow M$ such that for every $x \in$ int $N, d(f(x), g(x))<\varepsilon(x)$.

In dimension 4 very little is known (see [2] and [19] for partial results) both Resolution problem as well as General position problem are still open, while in dimensions $\geqq 5$ there also remain some questions, e.g.

\section{QUESTION 5.8. Does there exist a nonresolvable generalized 5-manifold?}

Also, there is still no written details available of the R.D. Edwards proof of the 5-dimensional case of Theorem (4.2) - the manuscript [21] deals only with the higher dimensions $(\geqq 6)$. It would be a worthwhile project to carefully rethink Edwards' argument for dimension 5 again and write it down, since there are some nontrivial engulfing problems one must know how to get around in this case.

Finally, the following is a related, very difficult problem from cohomological dimension theory, equivalent to the celebrated Cell-like mapping problem in dimension 4 (for more see the survey [26]):

QUESTION 5.9. Suppose that $f: M \rightarrow X$ is a cell-like map of a topological 4manifold $M$ onto a space $X . I s \operatorname{dim} X<\infty$ (equivalently, $\operatorname{dim} X=4)$ ? 
Note that by theorem of W.J.R. Mitchell, D. Repovš and E.V. Ščepin [27], $\operatorname{dim} X<$ $\infty$ if and only if $X$ has a certain kind of general position property, called the disjornt Pontryagin triples property.

Acknowledgements. This survey paper is based on a lecture presented at the Workshop on Topology and Geometry in Hanoi, Vietnam (25-31 March, 1993). The author wishes to acknowledge Professors Huynh Mui and Yukio Matsumoto for their kind invitation and the Vietnamese mathematicians for their great hospitality. Many thanks are also due to the Editor, Professor Mutsuo Oka for his cooperation (and patience) in getting this paper off the author's desk and into the print.

The preparation of the manuscript was mostly done during the several visits in 1993 to the Steklov Mathematical Institute, on the basis of the long-term joint research program (1991-1995) between the Slovenian Academy of Arts and Sciences and the Russian Academy of Sciences.

\section{REFERENCE}

[1] J.W. Alexander, An example of a simply connected surface bounding a region which is not simply connected, Proc. Nat. Acad. Sci. U.S.A. 10 (1924), 8-10.

[ 2 ] M. Best vina and J.J. Walsh, A 1-LCC Shrinking theorem in dimension 4, preprint in preparation, UCLA.

[ 3 ] R.H. Bing, The Kline sphere characterization problem, Bull. Amer. Math. Soc. 52 (1946), 644653.

[4] A. Borel, Ed., Seminar on Transformation Groups, Ann. of Math. Studies 46, Princeton Univ. Press, Princeton, 1960.

[5] K. Borsuk, Theory of Shape, Monogr. Mat. 59, PWN, Warsaw, 1975.

[6] M.V. Brahm, The Repovs Conjecture, Doctoral dissertation, Univ. of Texas, Austin, 1989.

[7] M.V. Brahm, Approximating maps of 2-manifolds with zero-dimensional nondegeneracy sets, Topol. Appl. 45 (1992), 25-38.

[ 8 ] M.G. Brin, Improving 3-manifold compactifications of open 3-manifolds, Houston J. Math. 4 (1978), 149-163.

[9] M. Brown, A proof of the generalized Schoenflies theorem, Bull. Amer. Math. Soc. 66 (1960), 74-76.

[10] J.L. Bryant, S.C. Ferry, W. Mio and S. Weinberger, Topology of homology manifolds, preprint, FSU, SUNY at Binghamton, IAS and Univ. of Chicago, 1992.

[11] J.L. Bryant and R.C. Lacher, Resolving zero-dimensional singularities in generalized manifolds, Math. Proc. Camb. Phil. Soc. 83 (1978), 403-413.

[12] J.W. Cannon, Taming codimension one generalized submanifolds of $S^{n}$, Topology 16 (1977), 323-334.

[13] J.W. Cannon, The recognition problem: What is a topological manifold?, Bull. Amer. Math. Soc. 84 (1978), 832-866.

[14] J.W. Cannon, Shrinking cell-like decompositions of manifolds: Codimension three, Ann. of Math. (2) 110 (1979), 83-112.

[15] R.J. Daverman, Products of cell-like decompositions, Topol. Appl. 11 (1980), 121-139.

[16] R.J. Daverman, Detecting the disjoint disks property, Pacif. J. Math. 93 (1981), 277-298.

[17] R.J. Daverman, Decompositions of Manifolds, Academic Press, Orlando, FL., 1986.

[18] R.J. Daverman and D. Repovš, A new 3-dimensional shrinking theorem, Trans. Amer. Math. 
Soc. 315 (1989), 219-230.

[19] R.J. Daverman and D. Repovš, General position properties that characterize 3-manifolds, Canad. J. Math. 44 (2) (1992), 234-251.

[20] R.J. Daverman and J.J. Walsh, A ghastly generalized n-manifold, mlinois J. Math. 25 (1981), 555-576.

[21] R.D. Edwards, Approximating certain cell-like maps by homeomorphisms, manuscript, UCLA, Los Angeles, 1977.

[22] M.H. Freedman, The topology of four-dimensional manifolds, J. Diff. Geom. 17 (1982), 357-453.

[23] W. Jakobsche and D. Repovš, An exotic factor of $S^{3} \times \mathbf{R}$, Math. Proc. Camb. Phil. Soc. 107 (1990), 329-344.

[24] R.C. Lacher, Cell-like mappings and their generalizations, Bull. Amer. Math. Soc. 83 (1977), 495-552.

[25] A. Marin and Y.M. Visetti, A general proof of Bing's shrinkability criterion, Proc. Amer. Math. Soc. 53 (1975), 501-507.

[26] W.J.R. Mitchell and D. Repovš, Topology of cell-like mappings, Proc. Conf. Diff. Geom. and Topol., Cala Gonone 1988, Suppl. Rend. Fac. Sci. Nat. Univ. Cagliari 58 (1988), 265-300.

[27] W.J.R. Mitchell, D. Repovš and E.V. Ščepin, On 1-cycles and the finite dimensionality of homology 4-manifolds, Topology 31 (1992), 605-623.

[28] R.L. Moore, Foundations of Point Set Theory, Amer. Math. Soc. Colloq. Publ. 13 Providence, RI., 1962.

[29] V.A. Nicholson, 1-FLG complexes are tame in 3-manifolds, Gen. Topol. Appl. 2 (1972), 277-285.

[30] F.S. Quinn, Ends of maps I, Ann. of Math. (2) 110 (1979), 275-331.

[31] F.S. Quinn, Resolutions of homology manifolds, and the topological characterization of manifolds, Invent. Math. 72 (1983), 267-284.

[32] F.S. Quinn, Erratum, Invent. Math. 85 (1986), 653.

[33] F.S. Quinn, An obstruction to the resolution of homology manifolds, Michigan Math. J. 34 (1987), 285-291.

[34] D. Repovš, The recognition problem for topological manifolds, Geometric and Algebraic Topology, J. Krasinkiewicz, S. Spiez and H. Toruńczyk, Eds., PWN, Warsaw, 1986, 77-108.

[35] D. Repovš, Detection of higher dimensional topological manifolds among topological spaces, Giornate di topologia e geometria delle varietà, Bologna 1992, Rend. Sem. Geom. Univ. Bologna, Bologna 1992, pp. 113-143.

[36] D. Repovš and R.C. Lacher, A disjoint disks property for 3-manifolds, Topol. Appl. 16 (1983), 161-170.

[37] D. Repovš and R.C. Lacher, Resolving acyclic images of nonorientable three-manifolds, Proc. Amer. Math. Soc. 90 (1984), 157-161.

[38] T.L. Thickstun, An extension of the loop theorem and resolution of generalized 3-manifolds with O-dimensional singular set, Invent. Math. 78 (1984), 161-222.

[39] J.J. Walsh, General position properties of generalized manifolds: a primer, Proc. Idaho State Topology Conf., 1985.

[40] R.L. Wilder, Topology of Manifolds, Amer. Math. Soc. Colloq. Publ. 32 Providence R.I., 1963. 
StekLov Mathematical InStitute

RUSSIAN ACADEMY OF SCIENCES

VAVILOVA STREET 42

117996 Moscow GPS-1, Russia

Permanent Address:

INSTITUTE FOR MATHEMATICS

Physics and Mechanics

UNIVERSITY OF LJUBLJANA

P.O.BOX 64, JADRANSKA 19

61111 Ljubluana, Slovenia

e-mail: dusan.repovs@uni-lj.si 